\title{
Design of APFC Panel for Industrial Application
}

\author{
${ }^{1}$ Deep Patel, ${ }^{3}$ Henil Patel, ${ }^{4}$ Krina Suhagiya \\ ${ }^{5}$ Rutvik Rohit, \\ 1,3,4,5 B.Tech Student, Electrical Engineering, \\ Indus University, \\ Ahmedabad, Gujarat, India.
}

\begin{abstract}
In modern days the power demand is increasing as the industrial load is increasing. There are various types of electrical and power electronic loads. These loads are fluctuating without manual interventions. These fluctuating loads can be stable with the use of a suitable capacitor. Majority of load are inductive in nature in industries. This inductive load consumes reactive power which affect the generation of the plant. Basically inductive load means lagging of power factor. To increase power factor there is a need of APFC Panel. Many industries use a lot of power from the grid but failed to utilize in an effective way. In many cases, consumer draws access to power than their sanctioned load. Therefore, the consumer has to pay a penalty. So, this penalty can be reduced by APFC Panel.
\end{abstract}

Keywords--- Power Factor, APFC Panel, Power factor correction, Inductive, Fixed capacitors, Penalty.

\section{I.INTRODUCTION}

In a current scenario, it has been observed that power is very prized for all and also demand of power is always high. The electric power system has grown in size and complexity with a huge number of interconnections to meet the increase in electric power demand. Most of the industrial plants are using the induction loads in infrastructure such as transformers and motors. Hence the use of more inductive load results in a lagging power factor i.e. the system power factor gets poor, due to access of reactive power consumed by inductive load such as induction motors which increases the reactive losses. Also, the reactive power consumption causes the reduction of voltage and power factor in networks. Power factor correction is the process of compensating a lagging current by a leading current through connecting capacitor to the supply. Practically, correcting power factor much nearer to the one may result in harmonic distortion. APFC or Automatic Power Factor control panels are mostly used in the improvement of power factor.

\section{II.NEED FOR POWER FACTOR CORRECTION}

- Varying power demand on the supply system.

- Power factor $(\cos \varphi)$ also varies as a function of the load requirements.

- Difficult to maintain a consistent power factor $(\cos \varphi)$ by use of fixed compensation.

- Leading power factor under light load condition.

- Automatically variation without manual intervention, the compensation to suit the load requirement.

- Increase available power

- Reducing the installation size

- $\quad$ Reducing the voltage drops on installation

\author{
${ }^{2}$ Dr. Sweta Shah \\ ${ }^{2}$ Assistant Professor, \\ Dept. of Electrical Engineering, \\ Indus University, \\ Ahmedabad, Gujarat, India
}

\section{III.PENALTY REDUCTION METHODS EXISTING METHODS \\ 1. Static Capacitors}

In the passage of time many methods have been used for power factor correction, one mainly prevalent method is that of a static capacitor bank. In this method, capacitors are normally known as a static capacitor induces a leading current and partly or completely neutralizes the lagging reactive component of load current. This raises the power factor of the load. For these loads the power factor capacitors can be connected in delta or star. Static capacitors are invariably used for power factor improvement in factories and industries.

\section{SYNCHRONOUS CONDENSER}

Asynchronous motor takes a leading current when overexcited and therefore behaves as a capacitor. When that category of a machine is coupled in parallel with the supply it takes a leading current which partly neutralizes the lagging reactive component of the load. Therefore the power factor is significantly improved. In power factor improvement by a synchronous condenser, the three-phase load takes current IL at low lagging power factor $\cos \phi \mathrm{L}$. The synchronous condenser takes a current Im which the voltage by a by an angle $\mathrm{pm}$. The resultant current $\mathrm{I}$ is the phasor sum of Im and IL and lags behind the voltage by an angle $\phi$. It is clear that $\phi$ is less than $\phi \mathrm{L}$ so that $\cos \phi$ is greater than $\cos \phi \mathrm{L}$. Thus the power factor is increased from $\cos \phi \mathrm{L}$ to $\cos \phi$. Synchronous condensers are generally used at large scale supply substations for power factor improvement.

\section{PHASE ADVANCERS}

They are used to improve the power factor of induction motors. Due to the fact that the stator winding draws exciting current which lags behind the supply voltage by $90^{\circ}$, induction motors have a low power factor. If the exciting ampere turns can be managed from some other A.C source then the stator winding will be relieved of exciting current and the power factor of the motor can be improved. This job is skilled by the phase advancer which is simply an AC exciter. The induction motor can be manufactured to operate on leading power factor like an over-excited synchronous motor. Phase advancers have an advantage because the exciting ampere-turns are supplied at slip frequency .therefore lag not KVAR drawn by the motor are considerably reduced. Phase advancers can be conveniently used where the use of synchronous motors is inadmissible. 
However, the primary disadvantage of phase advancers is that they are not economical for motors below 200HP.

\section{IV.PROPOSED METHOD}

For industrial purpose to improve the power factor, aPFC panel is used which includes capacitor with the detuned reactor. It will maintain the power factor and maximize the receving power. It contains APFCR relay as shown in figure 1. It is a microcontroller device which includes current control, voltage control senser and many more. And with the help of that, selection of capacitor will be done smoothly.



Figure 1:- SIEMENS APFCR Relay

\section{V.DESIGN METHODOLOGY}

Panel design comprising following steps:

1. GA Drawing

2. SLD Drawing

3. Schematic Wiring Diagram

4. Power and Control wiring Diagram

1. GA (General Arrangement)

GA requires following details to accomplish the panel design.

$>$ Details of transmitted data.

$>$ SLD with incoming and outgoing lines details.

$>$ The formation list for the components to be used.

$>$ The rating of the input lines for specific applications.

During this project, we measure the varied parameters (length, width, height) of panel and pieces of equipment. By using these parameters, we made a GA diagram of the APFC panel. It refers to the location of varied equipment and assemblies within the aggregated design of the electrical panel. It is a schematic representation of a corporal electrical panel design. Figure as

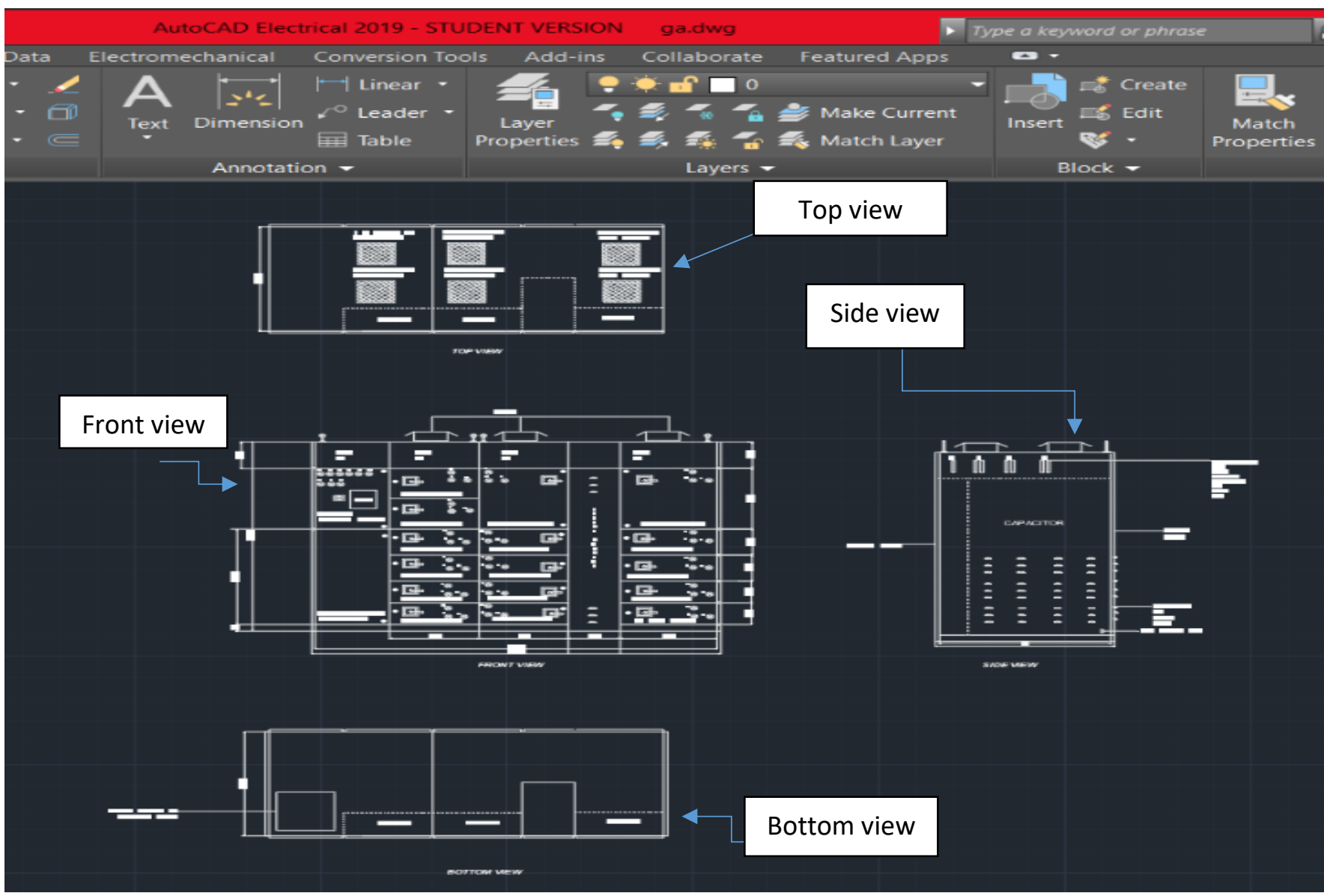




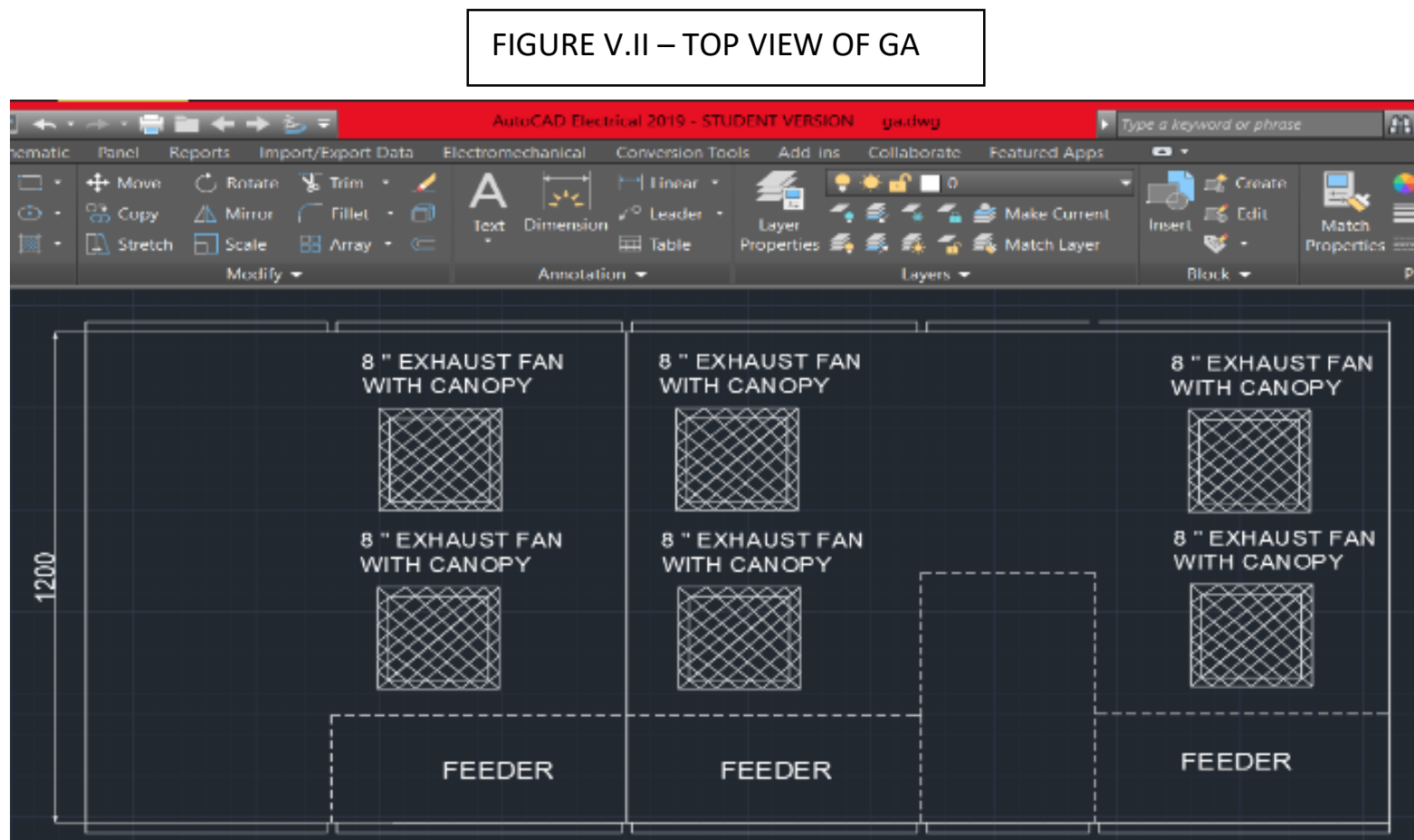

TOP VEW

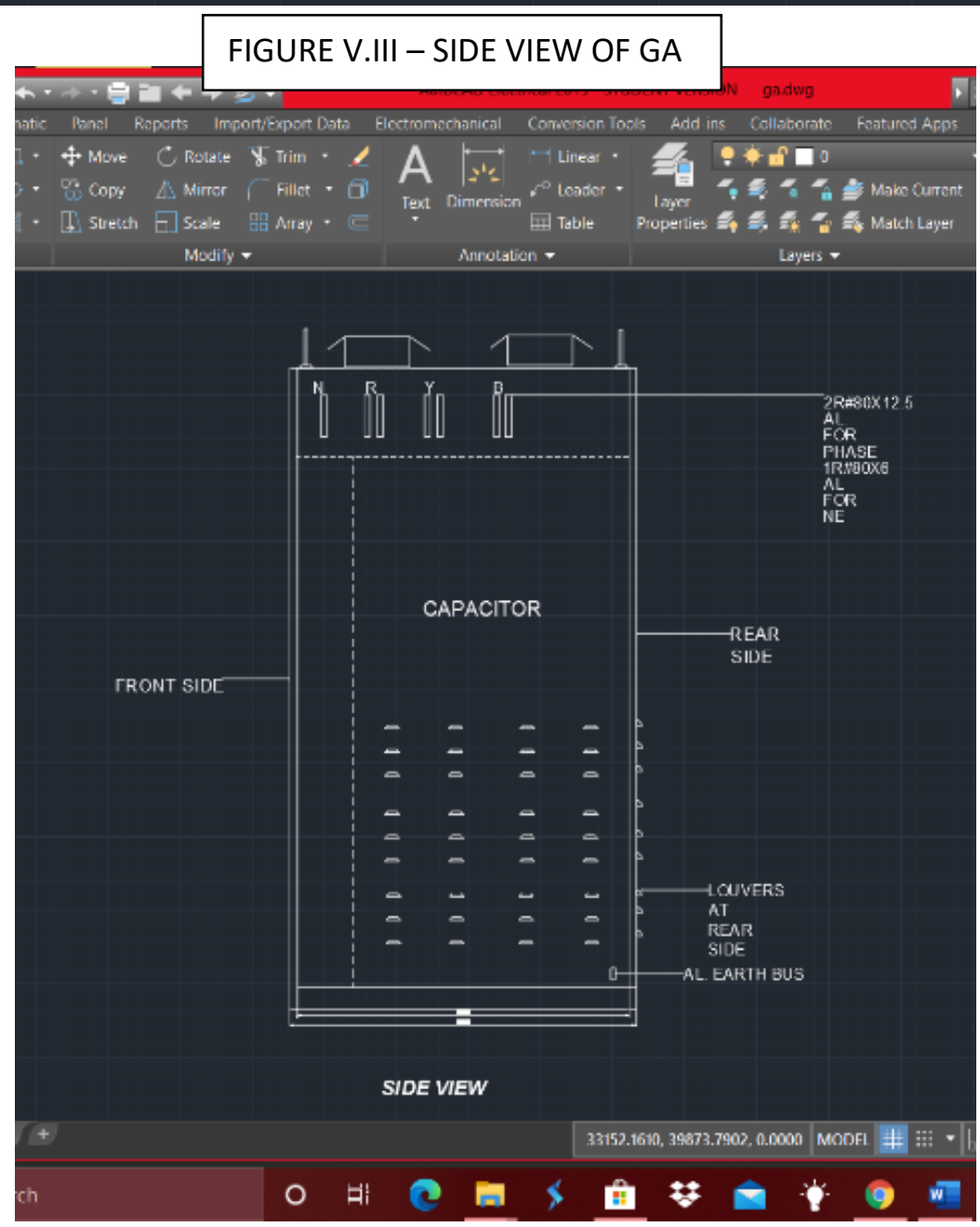



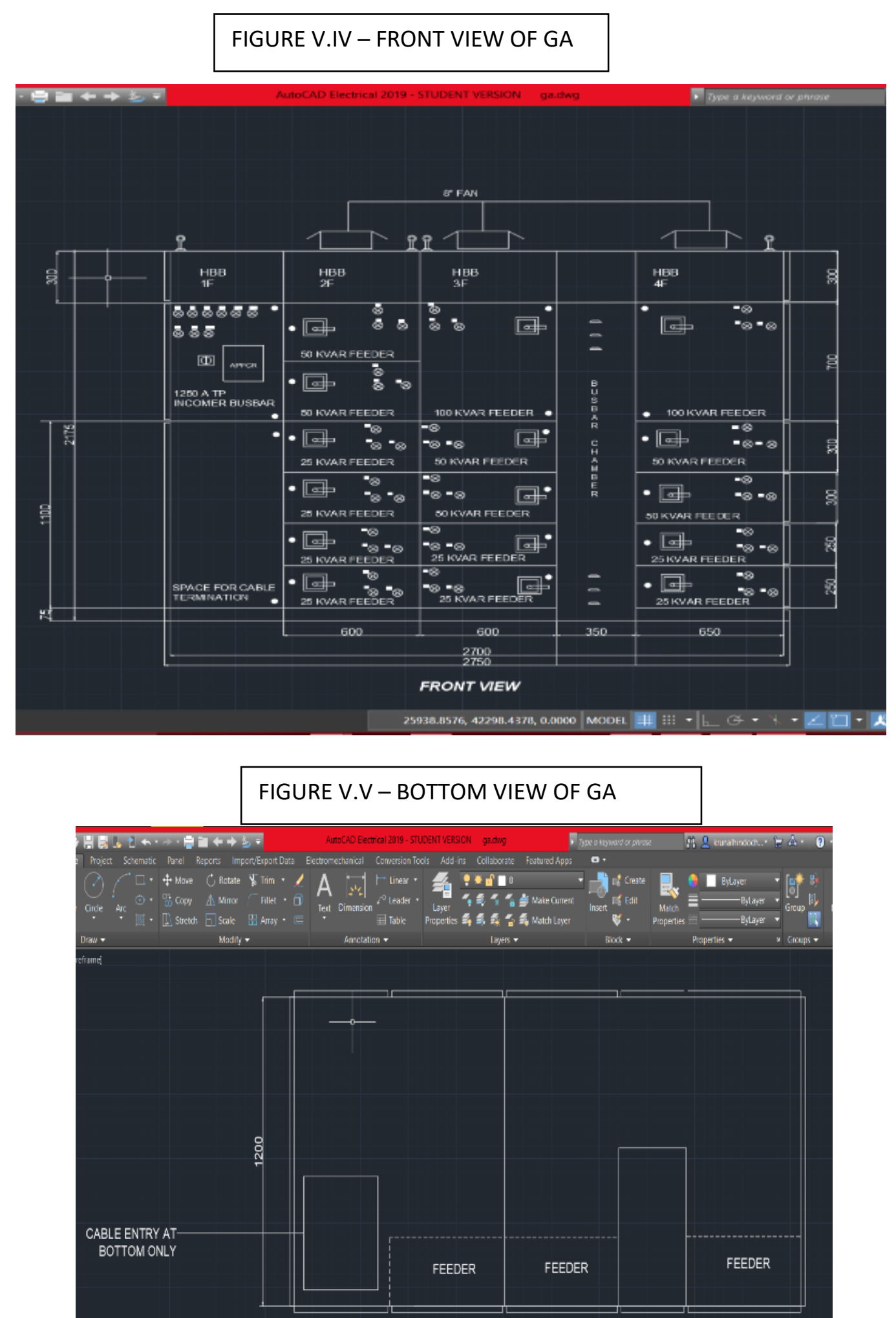
connected with $6 \mathrm{~A}$ single phase $\mathrm{MCB}$ for phase wire and with the neutral link for the neutral wire. In addition to all this another 3 indication lamp for ON, OFF, TRIP condition is provided. This 3 lamp is connected in parallel with the auxiliary contactor for tripping purpose. Along with that, a neutral link is connected with the neutral wire. Now as the supply is ON, the ON indication lamp will be ON and the other 2 lamp will be OFF at the same time. Similarly, during the OFF condition, the OFF indication lamp will be ON and the other will be OFF. Whenever during a short circuit or overcurrent or any other condition the TRIP indication lamp will get $\mathrm{ON}$ and the other 2 will be OFF.

\section{POWER AND CONTROL WIRING} DIAGRAM

In this figure, we can see that there are capacitor bank, inductor, power contactor, and fuse. This all are connected in series. In the normal condition, the power contactor is open. When power is $\mathrm{ON}$, the circuit becomes closed. There are two ways to control the circuit manual mode and auto mode. In the manual mode, it is operating manually and in auto mode it will be controlled by APFCR relay. There are manually start and stop push button. In

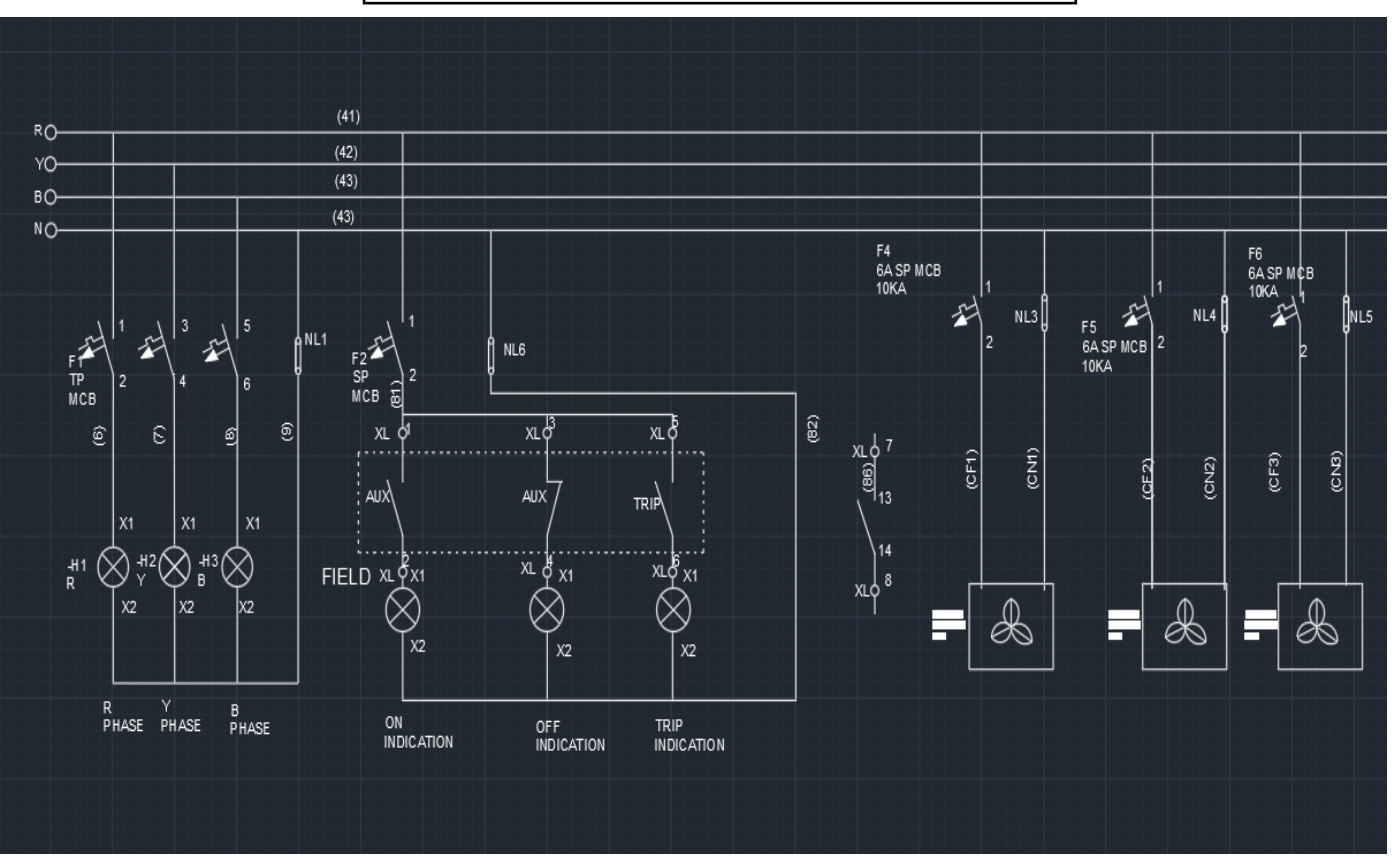

4. POWER AND CONTROL WIRING DIAGRAM

In this figure, we can see that there are capacitor bank, inductor, power contactor, and fuse. This all are connected in series. In the normal condition, the power contactor is open. When power is $\mathrm{ON}$, the circuit becomes closed. There are two ways to control the circuit manual mode and auto mode. In the manual mode, it is operating manually and in auto mode it will be controlled by APFCR relay. There are manually start and stop push button. In the intial condition stop push button is in the close condition, whereas start push button is in the open condition. When the power is ON in the circuit that time lamp blows ON. There is MCB to protect the control circuit from the short circuit and over-voltage condition. Figure as shown V.VIII the intial condition stop push button is in the close condition, whereas start push button is in the open condition. When the power is $\mathrm{ON}$ in the circuit that time lamp blows ON. There is MCB to protect the control circuit from the short circuit and over-voltage condition. Figure as shown V.VIII 







\section{VI.CONCLUSION}

It can be concluded that power factor correction techniques can be applied to the power system and due to that the system becomes stable and reliability of the system. In this research, capacity of shunt capacitor bank installed at $230 \mathrm{kV}$ line. Capacitor banks have generated reactive power and requirement of reactive power drawl from the system reduces it further reduces the corresponding amount of current in the line. Therefore applications of capacitor banks on substation, reduces the reactive power flow and reduce the losses in square proportion. In result, improvement in power factor from 0.5 to 0.94 in stage -9 . The APFC device helps to pull in high current drawn from the system and reduce charges on utility bills. A reduced power consumption results in lower greenhouse gas emissions and fossil fuel depletion by power stations and would benefit the environment.

\section{VII.REFERENCES}

1. International Journal OF Recent Technology and Engineering (IJRTE) ISSN: 2277-3878, Volume-2, Issue-4, September 2013.

2. https://download.schneider-electric.com

3. https://new.siemens.com/in/en/products/automation/industrialcontrols/7ug-monitoring-relays.html

4. http://www.electrosome .com

5. http://www.metroelectrician.com/uploads/1/1/0/9/110950405/control _panel_technical_guide.pdf

6. Asst. Prof. Smitha Paulose1, Ann Mary George2, Linu Jose3, Sruthi Harikumar4, "Reactive Power Compensation Using SVC" International Research Journal of Engineering and Technology (IRJET) Volume: 03 Issue: 06 | June-2016.

7. Alexander, C. K., \& Sadiku, M. N. (2007). Fundamentals of electric circuits. Boston: McGraw-Hill Higher Education.

8. "POWER FACTOR CORRECTION." www.nhp.com.au. NHP Catalogue- PFC 